\title{
An Educational Physics Laboratory in Mobile Versus Room Scale Virtual Reality - A Comparative Study
}

\author{
https://doi.org/10.3991/ijoe.v13i08.7371 \\ Johanna Pirker $\left.{ }^{\varpi}\right)$, Isabel Lesjak \\ Graz University of Technology, Graz, Austria \\ jpirker@iicm.edu \\ Christian Gütl \\ Graz University of Technology, Graz, Austria \\ Curtin University, Perth, Western Australia
}

\begin{abstract}
The emergence of new digital tools supporting immersive and engaging learning through Virtual Reality is opening up new paths for both distance, but also classroom learning. In this article we discuss the virtual physics laboratory "Maroon" and discuss experiences with Maroon in a cost-effective mobile setup with a mobile VR experience through Samsung GEAR and compare it with a more interactive VR experience in room-scale VR with HTC Vive. We describe a comparative evaluation of these two setups in order to identify chances and challenges of both setups. First results indicate more flexibility and portability with the mobile setup, while the room-scale setup profits of a highly interactive and hands-on experience. We discuss and compare the two setups based on immersion, engagement, presence, and motivation.
\end{abstract}

Keywords—virtual reality, immersion, physics education, engagement

\section{Introduction}

STEM education is still often received as a boring and not intuitive field and educators are challenged to find interesting and engaging ways to teach these concepts. In physics, interactive simulations and visualizations and virtual laboratories have been shown as promising methods to teach complex concepts and can enhance the understanding of these concepts [1][3][8] by making unseen concepts visible, stretching time, and conduct dangerous or even impossible experiments [2][13]. While understanding the concepts is crucial, current pedagogical models tend to involve many remote learning exercises. Such remote environments challenge the students to learn in a self-directed wa. Engagement and motivation are key elements of self-directed learning. Hence, the goal of digital and also blended learning experiences is to achieve student engagement, enthusiasm, and curiosity while teaching physical principles. Different authors have shown that immersive, playful, and interactive learning experiences in virtual laboratories or applications engage students more than compared to traditional methods [4][15]. Emerging virtual reality technologies open up new paths and possibilities for digital education with online and blended learning 
environments supporting such digital visualizations, simulations, and laboratory setups.

With the emergence of different virtual reality head-mounted displays (HMD) such as Oculus Rift or HTC Vive and also cost-effective mobile VR solutions (such as Samsung Gear VR, Google Cardboard), new possibilities for interaction and learning as well as novel application scenarios for virtual physics laboratories and interactive simulations have arisen. Different devices support different forms of interactions and learning solutions.

In this article we explore the potential of an interactive room-scale solution with the HTC Vive compared to the cost-effective mobile virtual reality setup through the Samsung Gear to create new possibilities for engaging in-class but also remote physics education. For this purpose, we have designed and implemented the physics laboratory Maroon in both mobile and room-scale VR to showcase different educational simulations and visualizations. The focus of the evaluation is to research interactive and immersive forms of education as improving engaging learning experiences. This work build on work published in [17] and extends it with a more detailed description of the study results.

This article aims on making the following contributions:

- Description of design and implementation of the physics laboratory Maroon in interactive and immersive mobile and room-scale VR setups

- The detailed description of two case studies investigating the potential and issues of mobile and room-scale virtual reality setups for learners with focus on immersion, engagement, and usability in a comparative study

The following section introduces background and related work in the field of interactive and engaging STEM education. In Section 3 we introduce the virtual physics laboratory Maroon. Section 4 discusses the two main experiments with the mobile and room-scale setup. Section 5 closes with a discussion about implications, potential, and further studies.

\section{Related Work}

In physics education a crucial element of the learning process is understanding various phenomena. In active learning approaches for physics education students, one way to teach abstract concepts is the interaction with these concepts through computer-based visualizations or animations, which make unseen phenomena visible and also allow small experiments [9][13]. Simulations have been shown by Wieman and Perkins as more effective, safe, and cost-efficient compared to traditional experiments [18]. Other successful virtual teaching methods include physics laboratories in digital form. Virtual or remote laboratories facilitate conducting dangerous, expensive, or even impossible experiments [6]. Such tools as part of an educational model either in a remote or in an in-class setup can make learning physics more effective, interesting, and engaging [18]. However, while these environments are often a successful learning tool they often fail to engage and convince students about the "fun" elements of this field. In a large-scale study with 306 participants Corter et al. [6] examined the learn- 
ing outcomes and student preferences for hands-on, remote, and simulated laboratories and found that learning outcomes after performing remote or simulated labs were as high or higher compared to hands-on labs. Students rated virtual labs as more convenient and reliable, but would prefer hands-on experiences. The feeling of physical presence in a lab was still rated as important factor of engaging laboratory experiences. In [11] the authors investigate various educational efforts in learning labs and conclude that such "alternative access modes must be considered pedagogical alternatives, rather than simply logistical conveniences" and point out the importance of a focus on pedagogical and interaction design. Especially in different VR environments, emotions and activities are perceived in a different way and it is crucial to consider different design aspects for the various VR technologies. A playful form of virtual laboratories has been tested in the field biotech education by Bonde et al. [4]. They tested a laboratory designed with gamification elements and found that this form of environment significantly increased the students learning outcomes and their performance compared with traditional teaching. In this digital and playful time, engagement, immersion, or even flow [7] are described ever more frequently as factors for creating interesting experiences. Immersion can be described as feeling of being part of the experience [5]. There is an ongoing discussion about the professional reality in remote and virtual laboratory experiences [12]. Adding immersion as main concept to the learning experience could be used to add new ways to create professional and interesting working and learning environments. The use of virtual reality headsets and technologies is a promising way to create a more immersive, engaging, and interactive environment. With the current efforts to produce VR headsets which are affordable for private users (e.g. PlayStation VR, Samsung Gear VR, HTC Vive), VR is also becoming more attractive as a tool to enhance classroom experiences. Several studies have looked into the potential of virtual reality (VR) for educational scenarios. In this paper, we introduce Maroon, an interactive immersive physics laboratory, integrated with (1) the interactive virtual reality technology HTC Vive, supporting in-room movement and a two-controller setup and (2) a mobile setup with the Samsung Gear VR.

\section{Maroon - The Immersive Physics Laboratory}

Maroon is an immersive physics laboratory designed to support interactive educational physics experiments and visualizations supporting different technologies such as virtual reality devices, mobile technologies, or web-based applications. Maroon is designed in the form of a laboratory and presents various educational experiments and simulations. In this paper we present two different forms of Maroon in a virtual reality setup with the goal to evaluate usability and user experience in VR and to measure factors such as engagement, immersion, and learning progress. Two distinct VR technologies were selected to base the comparative evaluation on. As a first device, the Samsung Gear VR was selected in order to evaluate mobile and more cost-effective environments (Maroon Mobile VR, see Fig. 1.). The HTC Vive was selected as a state-of-the-art interactive room scale VR technology (Maroon Room Scale VR, see Fig. 2.). 
Paper-An Educational Physics Laboratory in Mobile Versus Room Scale Virtual Reality - A Compara...

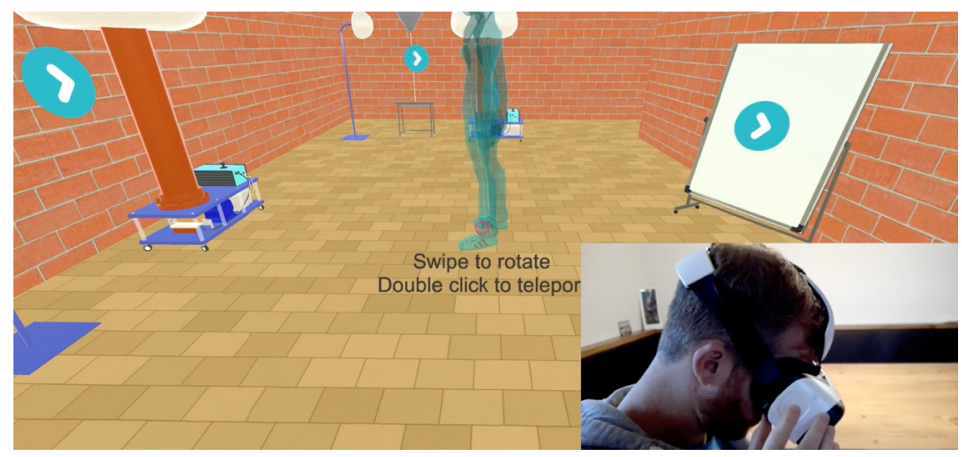

Fig. 1. The mobile VR experience with the Samsung Gear VR

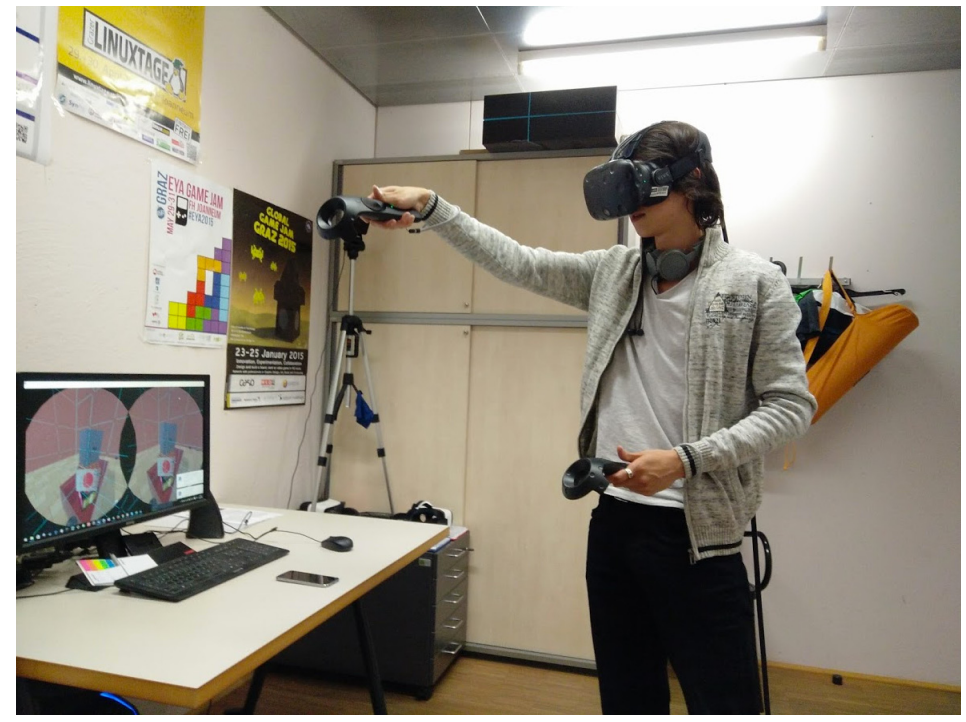

Fig. 2. The room-scale VR setup with the HTC Vive

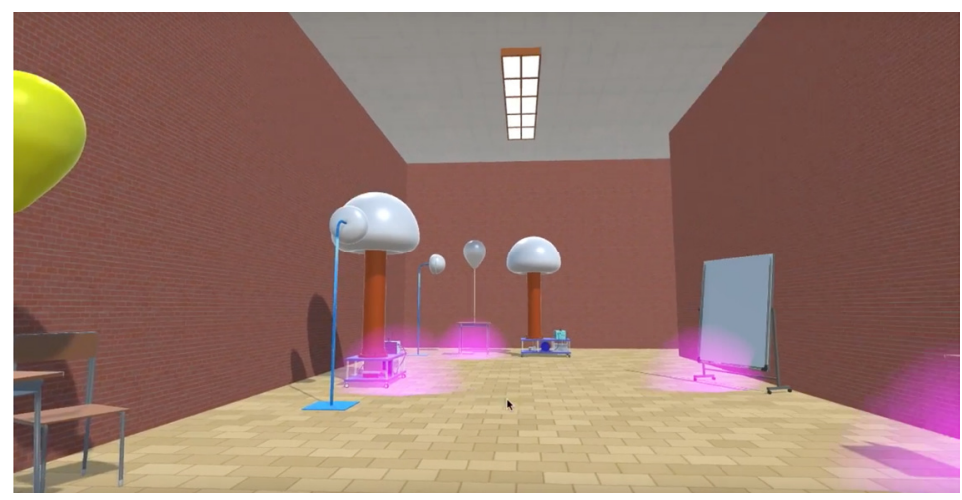

Fig. 3. The main room of Maroon 


\subsection{The Design}

As our research on Maroon includes two studies, the development of the laboratory was also done in two stages. In a first step, a prototype was developed. Unity supports stereoscopic rendering for different VR devices, including the Samsung Gear VR. For the HTC Vive, the official SteamVR 4 plugin and framework was used. This lab prototype was the design basis for the two VR variants. From originally six implemented simulations in the context of electromagnetism for this setup, only two were integrated in this initial prototype for the study. In our setup, users can experience VR in two distinct ways on two conceptually different devices: either through a mobile, more light-weight setup (Samsung Gear VR, using the Samsung Galaxy S6) or a more graphically rich, advanced room-scale system tracking both HMD and controllers (HTC Vive, using two controllers). In particular for user interaction, navigation, manipulation, and selection of UI elements with the virtual world, two different design approaches were chosen, considering various limitations and the different design of these two VR devices. The version of the immersive physics lab Maroon as introduced is designed to support both mobile VR systems such as Google Cardboard or Samsung Gear VR running on mobile phones as well as more advanced setups with room-scale VR such as the HTC Vive. The designed interaction with the environment and the experiments is mostly performed through gaze for the Samsung Gear VR and via controllers for the HTC Vive. Samsung Gear VR additionally provides possibilities to interact through touch and slide input, whereas the HTC Vive benefits from several buttons on both its tracked controllers which can be specifically programmed and also visually adapted for individual user actions. The navigation designs for the two VR alternatives are discussed in more detail in the following.

Navigation Design in Mobile VR. Given the Samsung Gear VR system with the smartphone inserted into a head-mounted gear, a real-life like user experience is achieved through a combination of eye gaze, a virtual avatar and a touchpad mounted on the side of the device, with user actions such as double tap, long press and swipe to rotate. Here, the user controls are mostly designed for gaze and tap interactions. An avatar (see Figure 1) is controlled with a gaze point to move through the laboratory. The avatar is always placed on the gaze point - the center of the screen - and can be moved by moving the gaze. Simulations can be started by moving the gaze cursor to the interaction button. Movement is designed as teleporting the avatar to different locations. Sliding (only supported by Samsung Gear VR) can be used optionally to rotate the character or to move specific controls (sliders) of experiments.

Navigation Design in Room Scale VR. In contrast, the HTC Vive system consists of a larger HMD connected to the PC as well as two additional controllers, which include a highly-sensitive touchpad and individually programmable buttons with haptic feedback for improved user interaction within virtual worlds. Each hardware element in the Vive setup is tracked by two base stations named lighthouses, thus eliminating the need for an avatar and further enabling the user to move around freely within the tracked area for a more immersive room-scale VR experience. Simulations are started by entering a portal-like object through button press on the controllers. Movement as in teleporting is achieved by pressing the touchpad on one of the con- 
trollers, which in turn acts like a pointer, as the user aims at the preferred target and displays a precise colored beam for visual orientation. Concerning the experimental setup, the main difference between the implementation for Samsung Gear VR and HTC Vive was the addition of interactable objects in the HTC Vive version and its lack of a virtual avatar which was instead implemented in the Samsung Gear version for better usability. By using several programmable controller buttons as well as touchpad press, HTC Vive users can benefit from further real-life like interaction possibilities. The necessity of a virtual avatar was not given for these since users carry both HMD and controllers which are being tracked by the lighthouse system.

Interactivities in the Lab The main interactivities integrated as experimental immersive setup for the study are as follows: a virtual laboratory room with different "stations" containing experiments or interactive activities (see Fig. 3), two experiments with a Van de Graaff Generator [10] which, combined with a balloon or a grounding device respectively, simulates electric fields while visualizing field lines as well a display of voltage and charge (see Fig. 3), and interactions with the controllers or the touchpad such as starting the experiment or teleporting. While the HTC Vive to some extend supports movement in the real room, the laboratory was designed as large-room experience; thus a teleporting functionality was necessary for both devices to reach all stations. Based on these interactivities 3-5 main educational experiences were included in our study setup of the virtual physics laboratory: (1) an experiment with a Van de Graaff Generator and a balloon, where charges, electric fields, and field lines can be visualized, (2) another experiment with a Van de Graaff generator and a movable grounding device where charges, electric fields, and field lines are visualized and a whiteboard with information and labeled pictures to explain the theory behind the Van De Graaff experiments. In order to showcase the manifold possibilities of user interaction with virtual objects using controller mechanisms, the HTC Vive version of this station additionally features an interactive playground with different textured objects such as throwable and grabbable cubes and metal balls. (4-only HTC) A triboelectric experiment with two rods and one balloon as well as a miniature version of the previous Van de Graaff experiment, however, this was only fully implemented for the HTC Vive test setup. Hence, to achieve more diversity in our experimental setting, this specific station was replaced by another station on the Samsung Gear VR version where it features a laptop with an interactive, feedback-supported quiz session in order to test the theoretical knowledge users should have gained with their practical hands-on walk-through of Maroon Mobile VR. (5-optional) Additionally, an accurate model of a Tesla transformator can be found by users as a hidden "easter egg" by further exploring the virtual laboratory world. In our research, these two conceptually different VR setups provide the frame for our implementation of the interactive immersive physics laboratory. Ultimately, the goal in developing these simulations is to let users act more or less the same way as they would act when placed in a real-life physics laboratory. As of now, users are - to some extent - able to immerse themselves into this world while being shielded from (visual) influences of their actual physical surrounding. As such, immersive $3 \mathrm{D}$ has shown to be a beneficial aid to present difficult concepts in physics, such as the effect of switching a Van De Graaff generator on and off. 


\section{$4 \quad$ Methods and Data}

We used two qualitative user studies with a total of 17 participants to study the two different setups with focus on getting insights into the overall experience and measuring (1) engagement, (2) immersion, (3) learning experience, (4) VR user experience, and (5) usability. In the first user study, we focused on evaluating the experience with the mobile VR setup ( 9 participants). In the second study, the interactive room scale VR experience was evaluated in comparison with the mobile VR experience by 8 participants, who each tested both setups.

\subsection{Material and Setup}

The VR setup for Samsung Gear VR consists of the following hardware components: the mobile HMD and the smartphone Samsung Galaxy S6. Figure 1 shows the Samsung Gear VR with the attached mobile phone. The setup for HTC Vive contains the HMD itself, cables and two base stations as well as two controllers. For a roomscale setup setting, we provided an area of about $2 \mathrm{~m} \times 2 \mathrm{~m}$. Furthermore, a powerful high-end hardware PC is necessary. Figure 2 illustrates this setup. A mobile VR setup was chosen in order to support a widely accessible and cost-effective way to interact with the laboratory, which could be used in classroom environments (e.g. guided by an instructor), or for self-regulated learning at home. The room-scale setup was chosen in order to assess the potential of more interactive hands-on experiences, which could be used at in-school learning laboratories.

\subsection{Method and Procedure}

For the first study with Samsung Gear VR, we first asked the participants to fill out a pre-questionnaire. The pre-questionnaire was used to get information about the participants experience with virtual experiences, VR technologies, and their expertise in physics. They were then briefly introduced to the system. After this they were asked to use the Maroon Mobile VR with the Samsung Gear VR. After the experience, the participants shortly described their impressions in form of an open dialog. Finally, they were asked to complete a post-questionnaire with 10 open-ended question about their experience and 20 single-choice questions with ratings on a Likert scale between 1 (fully disagree) and 7 (fully agree). In the other extended study with both devices, participants were required to fill out a short pre-questionnaire with standard personal background information, followed by a brief introduction to the experimental setup. Then the main goal was to complete consecutive tasks in the immersive lab Maroon, which were announced by the study moderator during the test run. Since we examine the differences and similarities of both devices, our eight test subjects were divided into two separate groups of four persons each, for the purpose of $\mathrm{AB} / \mathrm{BA}$ testing where users test both devices in reverse order. Specifically, four users tested the Vive first; whereas the other four tested the Samsung Gear VR first. After each single run, users completed a corresponding post-questionnaire containing 19 standardized questions from the Game Engagement Questionnaire (GEQ, [5]) to 
measure the level of engagement based on absorption, flow, presence, and immersion, as well as ten open-ended questions on the experience and 20 single-choice questions with ratings on a Likert scale between 1 (fully disagree) and 7 (fully agree). For a comparative evaluation, all subjects had to complete a "combined" post-questionnaire with open-ended questions about their experience on both devices at the end of the experiment.

\subsection{Participants}

The two case studies described in this section have been conducted separately and independent from each other, i.e. there is no overlap between the two study groups; one of which tested Maroon Mobile VR only (9 subjects), while the other group (8 subjects) tested Maroon Mobile VR in comparison with Maroon Room Scale VR.

Experiment 1. In the first study, 9 students (2f) between 23 and 27 (AVG=24.78, $\mathrm{SD}=1.47$ ) tested Maroon Mobile VR. All students were in the field of computer science or electrical engineering and rated their experience with computers very high. 6 students rated their selves on a Likert scale between 1 (not at all) and 5 (fully agree) also as very experienced in the usage of video-games ( $\mathrm{AVG}=4.11, \mathrm{SD}=1.17), 8$ like playing video games. All of them rated themselves as not very experienced in the usage of VR ( $\mathrm{AVG}=1.78, \mathrm{SD}=0.97) .7$ had heard of mobile VR devices before, 4 have used Google Cardboard, 5 the Samsung Gear VR. Rating their physics expertise, the results were very mixed $(\mathrm{AVG}=2.89, \mathrm{SD}=1.05)$.

Experiment 2. In the second study, 8 (1f) participants were asked to test the mobile (Maroon Mobile VR) as well as the interactive physics lab (Maroon Room Scale VR). 7 are very experienced in the use of computers $(\mathrm{AVG}=4.38, \mathrm{SD}=1.41)$, only 2 in the usage of video-games $(\mathrm{AVG}=3, \mathrm{SD}=1.2)$, and only 1 in $\mathrm{VR}(\mathrm{AVG}=2.25$, $\mathrm{SD}=1.39$ ). 4 have used a mobile VR setup before, nobody the HTC VIVE. 7 rated their physics knowledge a 3 or below $(A V G=2.63, S D=0.92)$. In the following sections we discuss different aspects of the outcomes from the post-questionnaires. The individual aspects will be mainly described by including outcomes of the questionnaire and direct quotes from answers describing the students' impressions and experiences.

\subsection{Comparing Mobile vs. Room-Scale VR Experiences}

In the second user study, eight participants tested both, the mobile VR setup on the Samsung Gear, as well as the room-scale VR setup on the HTC Vive. The comparison of these two different VR experiences was evaluated through the Game Engagement Questionnaire (GEQ), which all participants had to complete after finishing the experiment. An overview of the results for each GEQ statement is shown in Table 2. There are a few significant differences in the average value results for mobile and roomscale VR setups. Users rated the room-scale VR experience on the HTC Vive as (feeling) more real $(\mathrm{AVG}=3.25, \mathrm{SD}=0.89)$ than the mobile $\mathrm{VR}$ experience on the Samsung Gear $(\mathrm{AVG}=2.5, \mathrm{SD}=0.53)$. Furthermore, users felt more like losing track of time 
while using the HTC Vive $(\mathrm{AVG}=3.375, \mathrm{SD}=1.6)$. In contrast, using the Samsung Gear made users feel more different $(\mathrm{AVG}=3.25, \mathrm{SD}=1.49)$.

As it can be seen in Table 2, each statement in the GEQ can be assigned to one of the four main categories which contribute to game engagement: presence, absorption, flow, and immersion. An overview of the overall results for each category is listed in Table 1. The HTC Vive scores slightly better than the Samsung Gear in each of the four categories.

Table 1. Comparison of average and standard deviation between GEQ categories for HTC Vive and Samsung GEAR in Experiment 2

\begin{tabular}{lcccc}
\hline & \multicolumn{3}{c}{ HTC VIVE } & \multicolumn{3}{l}{ SAMSUNG GEAR } \\
\hline Category & AVG & SD & AVG & SD \\
Presence & 2.59 & 0.18 & 2.41 & 0.36 \\
Absorption & 2.4 & 0.098 & 2.28 & 0.07 \\
Flow & 2.51 & 0.18 & 2.278 & 0.30 \\
Immersion & 2.88 & 1.36 & 2.63 & 1.51 \\
\hline
\end{tabular}

Table 2. Comparison of average and standard deviation between GEQ statements for HTC Vive and Samsung GEAR in Experiment 2

\begin{tabular}{|c|c|c|c|c|c|}
\hline \multirow[b]{2}{*}{ GEQ Statement } & \multirow[b]{2}{*}{ Category } & \multicolumn{2}{|c|}{ HTC VIVE } & \multicolumn{2}{|c|}{ SAMSUNG GEAR } \\
\hline & & $A V G$ & $S D$ & $A V G$ & $S D$ \\
\hline 1 I lose track of time & Presence & 3.375 & 1.6 & 2.75 & 1.7 \\
\hline 2 Things seem to happen automatically & Presence & 2 & 1.2 & 2.25 & 1.04 \\
\hline 3 I feel different & Absorption & 2.875 & 1.64 & 3.25 & 1.49 \\
\hline 4 I feel scared & Absorption & 1.5 & 1.41 & 1.5 & 1.41 \\
\hline 5 The game feels real & Flow & 3.25 & 0.89 & 2.5 & 0.53 \\
\hline 6 If someone talks to me, I don't hear them & Flow & 1.5 & 1.07 & 1.625 & 1.41 \\
\hline 7 I get wound up & Flow & 2.625 & 1.41 & 2.375 & 1.51 \\
\hline 8 Time seems to kind of stand still or stop & Absorption & 2.875 & 1.55 & 2 & 1.31 \\
\hline 9 I feel spaced out & Absorption & 2.25 & 1.49 & 2.25 & 1.49 \\
\hline 10 I can't tell that I'm getting tired & Flow & 2.5 & 1.31 & 2.25 & 1.38 \\
\hline 11 Playing seems automatic & Flow & 2.5 & 1.31 & 2.25 & 1.28 \\
\hline 12 My thoughts go fast & Presence & 2.5 & 1.31 & 2 & 0.93 \\
\hline 13 I lose track of where I am & Absorption & 2.5 & 1.41 & 2.375 & 1.41 \\
\hline 14 I play without thinking about how to play & Flow & 3 & 1.41 & 2.625 & 1.06 \\
\hline 15 Playing makes me feel calm & Flow & 3 & 1.2 & 2.875 & 1.13 \\
\hline 16 I play longer than I meant to & Presence & 2.5 & 1.51 & 2.625 & 1.51 \\
\hline 17 I really get into the game & Immersion & 2.875 & 1.36 & 2.625 & 1.51 \\
\hline 18 I feel like I just can't stop playing & Flow & 2.625 & 1.19 & 2.125 & 0.99 \\
\hline 19 I don't answer when someone talks to me & Flow & 1.625 & 1.06 & 1.875 & 1.46 \\
\hline
\end{tabular}




\subsection{Experiencing Immersion and Engagement}

In the first user study testing the mobile VR setup on the Samsung Gear, most of the participants said they find learning in this manner more engaging $(A V G=5.67$, $\mathrm{SD}=1.80)$ and fun $(\mathrm{AVG}=5.56, \mathrm{SD}=1.88)$. When being asked if they find it engaging and motivating, most of them agreed: "very motivating way of demonstrating stuff". The lack of content and variety was mentioned as a drawback here: "Not yet, but I can see how the concept would be engaging once more variety exists." When asked what they liked about the system, immersive and three-dimensional characteristics were mentioned in particular: "Immersion makes me remember stuff better". Overall, the VR experience was received very positive and described as very immersive by the participants.

In the second user study, we compared factors contributing to engagement such as presence, absorption, flow, and immersion (as part of the GEQ) between the roomscale VR experience on the HTC Vive setup and the mobile VR setup on the Samsung Gear. As already described in Section 4.4 the interactive version of Maroon VR on the HTC Vive achieves only slightly better results in all four categories of the GEQ.

However, immersion and engagement were not only evaluated via the 19 standardized questions of the GEQ, but also through 20 single-choice questions with Likertscale ratings and 10 open-ended questions on the experience. Findings of these two question formats are presented below: Participants interacting with the Maroon VR on the HTC Vive found this way of learning more engaging $(\mathrm{AVG}=5.375, \mathrm{SD}=1.77$ ) and more fun $(\mathrm{AVG}=5.375, \mathrm{SD}=2.07)$ than on the Samsung Gear, which participants found slightly less engaging $(\mathrm{AVG}=5.125, \mathrm{SD}=1.64)$ and less fun $(\mathrm{AVG}=4.75$, $\mathrm{SD}=1.83$ ) for learning.

When being asked the question "Do you find it engaging and motivating?" , comments on the HTC Vive highlighted the aspect of fun: "Yes it is quite engaging and motivating to use. Learning seems much more fun this way." and "Yes, as said: The simplest things become fun in Virtual Reality". One person thought about potential effects of nausea: "Quite definitely. Though I wonder if nausea might have a negative impact on learning experience". Remarks on the Samsung Gear included very positive statements such as "Definitely. Playing and instant feedback (sth is moving, machine working, flashes striking..) catches my interest and makes me want to find out more." as well as statements indicating a stronger bias towards HTC Vive: "yes, maybe more if I didn't know the vive" as well as "A bit less than with the Vive". In fact, two users of this second study did not find the mobile VR experience engaging and motivating at all.

\subsection{Experiencing Learning}

In the first study, on a Likert scale between 1 (not at all) and 7 (fully agree) most of the people questioned said they would like to learn with Maroon Mobile VR $(\mathrm{AVG}=4.67, \mathrm{SD}=1.87)$ and feel that the content is easier to understand $(\mathrm{AVG}=4.89$, $\mathrm{SD}=1.83)$ and more motivating than ordinary exercises $(\mathrm{AVG}=4.89, \mathrm{SD}=1.96)$. However, the environment inspired only a few to learn more about physics $(A V G=2.67$, 
$\mathrm{SD}=1.41$ ). When we asked them if they would use it for learning, all but one of the participants were positive about this idea. Many positive comments mentioned the experimentation and visualization of usually unseen things: "I would use it immediately for my mechanical engineering studies, because it is an advantage to see and rotate the machines in a 3D space; also it can be an advantage when learning about dangerous machines: one can still see everything without a distance". It was also mentioned that they would like to use the immersive lab as supplement for regular learning $(\mathrm{AVG}=5.67, \mathrm{SD}=1.41)$. The students of the evaluation group would rather like to use Mobile VR in a class-room environment ( $\mathrm{AVG}=4.78 ; \mathrm{SD}=2.11$ ) than at home ( $\mathrm{AVG}=4.22, \mathrm{SD}=1.99)$. "There are a few elements missing that would produce a good learning environment for me. The first thing are explanations. If someone learns about the illustrated concepts beforehand (maybe in a class), the game could certainly help with that, but it is far from a standalone learning tool right now.". Concerns using this system for learning include the topic choice ("It's good for demonstrating something, maybe not as good for learning facts etc., because you can't for example take notes etc.") and additional overhead. The VR aspect was very well received for learning. Participants thought it was engaging to see the physics simulations with the VR glasses $(\mathrm{AVG}=5.78, \mathrm{SD}=1.92)$ and also a bit more engaging than without VR ( $\mathrm{AVG}=5.44, \mathrm{SD}=1.42)$ as is reflected in the statement "learning with VR is gonna be awesome and I never thought about what happens to a balloon if we place him between a Tesla-coil and a grounder. Funny".

For the interpretation of results from the second user study, it is important to consider that each of the 8 study participants had experienced and tested the physics laboratory with both VR devices, albeit in different order via A/B, B/A testing. Specifically, the first group of 4 subjects tested the HTC Vive first, whereas the second group of another 4 subjects tested the Samsung Gear first. In the second user study, most participants stated that they would like to learn with Maroon Room-Scale VR on the HTC Vive $(\mathrm{AVG}=5.125, \mathrm{SD}=2.10)$ and think that the content is easier to understand $(\mathrm{AVG}=5.5, \mathrm{SD}=1.41)$ and more motivating than ordinary exercises $(\mathrm{AVG}=5.375, \mathrm{SD}=1.41)$. However, only a few participants would agree to buy the VR glasses and download the Immersive Physics Lab for use at home $(A V G=3$, $\mathrm{SD}=2.07$ ).

Concerning learning with the Samsung Gear, users would not like to learn with Maroon Mobile VR as much $(\mathrm{AVG}=4.625, \mathrm{SD}=2.39)$. This sentiment is also reflected in the fact that users of the Samsung Gear did not find the content as easy to understand $(\mathrm{AVG}=4.625, \mathrm{SD}=1.92)$ and as motivating $(\mathrm{AVG}=4.25, \mathrm{SD}=1.67)$ as on the HTC Vive.

When being asked Would you use it for learning?, answers about Maroon VR on HTC Vive were quite varied, ranging from very positive like "Absolutely! It is very fun and makes learning an activity to look forward to" to rather critical such as "Not really, the "game and fun factors" are too high so it could be hard to focus on the important things for learning." and "Depends on the subject. For something like physics, where there is actually something to see yes. Others, like mathematics I am not sure whether this would be helpful". Users definitely like the fun part, but are not fully convinced about the learning aspect. In contrast to the first user study, only half 
of the test users of this second study would like to use the Samsung Gear for learning, after also having experienced VR on the HTC Vive. Users' answers mentioned drawbacks such as user interaction ("the handling takes away a bit of the joy of exploring the vr world") and perceived effort versus usefulness ("not worth the effort, because the visualization could be done on the computer as well", "Depends on the subject again, physics yes other subject I am not convinced.").

When users were asked whether they find Maroon VR good for learning, both the HTC Vive and the Samsung Gear version received mixed responses. With Maroon Room Scale VR, users liked the possibility of actually exploring the virtual world up close and in real-time ("I can imagine it to be very good for learning. Seeing the Reactions in Real Time and up close gives a better understanding of many physical processes which are happening") but also remarked the necessity of providing further theoretical background ("If one would be more familiar with this topic area, then yes, it would probably be good for learning. Without that, one felt a bit helpless without knowing what this simulation is supposed to show").

For Maroon Mobile VR, users would generally like to use it for learning but again saw the limited handling and low resolutions as a drawback: "its ok, but the limited handling takes some of the motivation for engaging with the environment away" and "The experiments for sure. The board with the lecture slides was too low resolution to recognize it for a means to convey actual content. Reminded me much of FPS games like HL, Doom3 where text was merely a deco on virtual computer consoles." Again, users see this as an additional tool to supplement or deepen existing knowledge about physics ("Maybe it could be used as additional tool for better understanding of experiments." "maybe if I knew more about the field of physics").

\subsection{Experiencing Usability and User Experience}

In the first study, usability and user experience differed from person to person. While some of the people had no issue with the controls and the interface, others had problems here, especially with learning the movements. Minor usability issues were mentioned. These included in particular the unusual movement (teleporting instead of walking; how to turn the avatar) and interactions (e.g. clicking twice on the door to exit a simulation instead just once). "Moving in the environment was not very intuitive, but worked well. The UI was not very hard to figure out." Additionally, the idea to give more feedback on interaction possibilities was mentioned "I wished for some visual feedback on what's clickable. I wasn't sure what I can click and what not so I clicked around quite a lot."

Findings for the second study are as follows: With the Samsung Gear VR, most study participants had a good overall impression. The main usability issues expressed by users were possibilities for interaction and movement, including a mention of cybersickness ("I'd like more options for interacting with the generator devices e.g. Fiddle around with the equipment, find out what it can do. Experiencing the VR environment was a bit unsettling sometimes. Sensory input from the natural environment mismatched what I saw in VR. Think, I felt slightly dizzy. The teleport for moving around gave me a punch in the stomach the first few times. Maybe the radical change 
of scenery in front of my eyes, but no physical movement whatsoever.") as well as low quality of graphics: "The text on the whiteboard and on the computer was very hard to read (maybe because of bad resolution?); movement not intuitive and a bit complicated."

With the HTC Vive, study participants enjoyed in particular the interaction with objects as well as the high graphics resolution: "I liked that I can actually touch and move things with my hands. Graphics are awesome too." Again, a feeling of dizziness characteristic for cybersickness was reported by some users: "After some time $>20$ minutes, I started feeling a noticeable dizziness and a hint of nausea (but nothing serious)." Other drawbacks remarked by students include the handling of controllers ("In the beginning, one needs a certain time to get used to it (which buttons are used for which action). However, controllers are definitely more convenient than the trackpad on the headset of Samsung Gear VR. Nevertheless, in the long term it might not be pleasant to always have to hold two extra things in the hands. ") and the limitations within the physical room: "Being able to grab and move things was great. Enjoyed that much. That I could move my body and was not confined to a chair was great. I grew a bit weary of the hitting something in the real world. Always checking the confines of my personal physical movement space." Some users also came up with suggestions for additional features such as collaboration between students and museum-like experiences within the lab: "Maybe it could fun to learn with others in VRs, but only as additional tool." "Information could be given to the Exhibitions. Either in form of a Text or a Museum-like Announcer voice. Actually a virtual Museum would be quite exciting."

\section{$5 \quad$ Discussion and Conclusion}

In this article, we have described and investigated two different forms of immersive learning experience in virtual reality environments for physics education. The goal was a exhaustive comparison between learning experiences in a virtual physics laboratory with cost-effective mobile VR technologies and with interactive room scale VR setups. First results indicate positive experience in both virtual reality experiences with the immersive physics laboratory "Maroon VR". Learning in such an immersive environment was described as engaging and interesting experience. Results also indicate that students would be in favor of using such technologies for learning and that they find it more engaging and also effective when compared with traditional learning scenarios. With this work we have shown the potential of emerging immersive and interactive technologies to become an integral part of future inclassroom learning. Many participants would recommend the use of virtual reality learning experience as supplement to in-class learning models than using it as a standalone application to learn in a self-regulated way at home.

Mobile VR setups such as experiences with the Samsung Gear VR or the Google Cardboard provide cost-effective, dynamic, and mobile learning experiences and can be easily set up for in-class learning experiences. One way to introduce virtual reality learning experiences in educational settings is to extend active learning strategies with 
short digital mobile VR experiences as part of the class room setting. Additionally, mobile virtual reality scenarios which support networked capabilities can be used to show all students experiences such experiments, visualizations, or simulations at the same time while the teachers guides through the experience.

Compared to mobile VR, room scale VR setups are more cost-intensive, need specific hardware and a lot of space, and only support a limited amount of users at the same time. However, many users feel more immersed and engaged by interactive room scale setups. Additionally, the controllers give users more possibilities to interact with experiments. This often creates more interesting hands-on experiences. Such virtual reality setups could be used in addition to classroom scenario, for instance as part of provided self-directed learning labs.

\section{Acknowledgment}

We would like to thank John Winston Belcher from the Department of Physics Massachusetts Institute of Technology for supporting this research. The Maroon projects is a research project at Graz University of Technology: gamelabgraz. com/maroon/. We thank all people who are and were involved in the development process. We would also like to express our gratitude and appreciation to Philip $\mathrm{H}$. Bailey for his constant (also last minute) support.

\section{$7 \quad$ References}

[1] Adams, W.K., Reid, S., LeMaster, R., McKagan, S.B., Perkins, K.K., Dubson, M., Wieman, C.E. (2008). A study of educational simulations part 1-engagement and learning. Journal of Interactive Learning Research 19(3), 397

[2] Aldrich, C. (2009). Learning online with games, simulations, and virtual worlds: Strategies for online instruction, vol. 23. John Wiley \& Sons

[3] Bell, R.L., Smetana, L.K. (2008). Using computer simulations to enhance science teaching and learning. National Science Teachers Association 3, 23-32

[4] Bonde, M.T., Makransky, G., Wandall, J., Larsen, M.V., Morsing, M., Jarmer, H., Sommer, M.O. (2014). Improving biotech education through gamified laboratory simulations. Nature biotechnology 32(7), 694-697 https://doi.org/10.1038/nbt.2955

[5] Brockmyer, J.H., Fox, C.M., Curtiss, K.A., McBroom, E., Burkhart, K.M., Pidruzny, J.N. (2009). The development of the game engagement questionnaire: A measure of engagement in video game-playing. Journal of Experimental Social Psychology 45(4), 624-634 https://doi.org/10.1016/j.jesp.2009.02.016

[6] Corter, J.E., Nickerson, J.V., Esche, S.K., Chassapis, C., Im, S., Ma, J. (2007). Constructing reality: A study of remote, hands-on, and simulated laboratories. ACM Transactions on Computer-Human Interaction (TOCHI) 14(2), 7 https://doi.org/10.1145/1275511.1275513

[7] Csikszentmihalyi, M., Csikszentmihalyi, I.S. (1992). Optimal experience: Psychological studies of flow in consciousness. Cambridge university press

[8] Dori, Y.J., Belcher, J. (2005). How does technology-enabled active learning affect undergraduate students' understanding of electromagnetism concepts? The Journal of the Learning Sciences 14(2), 243-279 https://doi.org/10.1207/s15327809jls1402 3 
Paper-An Educational Physics Laboratory in Mobile Versus Room Scale Virtual Reality - A Compara...

[9] Dori, Y.J., Hult, E., Breslow, L., Belcher, J.W. (2007). How much have they retained? making unseen concepts seen in a freshman electromagnetism course at mit. Journal of Science Education and Technology 16(4), 299-323 https://doi.org/10.1007/s10956-007$\underline{9051-9}$

[10] International, O.: Van de graaff generator (2014), http://research.omicsgroup. org/index.php/Van_de_Graaff_generator

[11] Lindsay, E., Good, M. (2006). Virtual and distance experiments: Pedagogical alternatives, not logistical alternatives. In: American society for engineering education. pp. 19-21

[12] Lowe, D., Murray, S., Lindsay, E., Liu, D., Bright, C. (2008). Reflecting professional reality in remote laboratory experiences. In: Proc. Intl Conf. Remote Eng. and Virtual Instrumentation (REV08)

[13] Lunce, L.M. (2006). Simulations: Bringing the benefits of situated learning to the traditional classroom. Journal of Applied Educational Technology 3(1), 37-45

[14] Olson, S., Riordan, D.G. (2012). Engage to excel: Producing one million additional college graduates with degrees in science, technology, engineering, and mathematics. report to the president. Executive Office of the President

[15] Pirker, J., Berger, S., Gütl, C., Belcher, J., Bailey, P.H. (2012). Understanding physical concepts using an immersive virtual learning environment. Proceedings of the 2nd European Immersive Education Summit, Paris pp. 183-191

[16] Pirker, J., Gütl, C. (2015). Educational gamified science simulations. In: Gamification in Education and Business, pp. 253-275. Springer https://doi.org/10.1007/978-3-319-10208$\underline{5 \quad 13}$

[17] Pirker, J., Lesjak, I. Parger, M., Gütl, C. (2017). An Educational Physics Laboratory in Mobile Versus Room Scale Virtual Reality - A Comparative Study. In Remote Engineering and Virtual Instrumentation (REV).

[18] 20. Wieman, C., Perkins, K. (1995). Transforming physics education. Physics today 58(11), 36 (2005) https://doi.org/10.1063/1.2155756

[19] 21. Windschitl, M.A.: Using computer simulations to enhance conceptual change: The roles of constructivist instruction and student epistemological beliefs

\section{Authors}

Johanna Pirker (corresponding author) is researcher at Graz University of Technology, Institute for Interactive Systems and Data Science, Inffeldgasse 16c, Graz Austria.

Isabel Lesjak is with Institute for Interactive Systems and Data Science at Graz University of Technology, Inffeldgasse 16c, Graz Austria.

Christian Gütl is Associated Professor at Graz University of Technology in Austria and Adjunct Research Professor at Curtin University of Technology in Perth, WA.

This article is a revised version of a paper presented at the International Conference on Remote Engineering \& Virtual Instrumentation (REV2017), held in New York, NY, USA, March 2017. Article submitted 27 June 2017. Published as resubmitted by the authors 30 july 2017. 